\title{
Tonic Descending Facilitation from the Rostral Ventromedial Medulla Mediates Opioid-Induced Abnormal Pain and Antinociceptive Tolerance
}

\author{
Todd W. Vanderah, Nova M. H. Suenaga, Michael H. Ossipov, T. Philip Malan Jr, Josephine Lai, and \\ Frank Porreca
}

Departments of Pharmacology and Anesthesiology, University of Arizona, Tucson, Arizona, 85724

\begin{abstract}
Many clinical case reports have suggested that sustained opioid exposure can elicit unexpected, paradoxical pain. Here, we explore the possibility that (1) opioid-induced pain results from tonic activation of descending pain facilitation arising in the rostral ventromedial medulla (RVM) and (2) the presence of such pain manifests behaviorally as antinociceptive tolerance. Rats implanted subcutaneously with pellets or osmotic minipumps delivering morphine displayed time-related tactile allodynia and thermal hyperalgesia (i.e., opioid-induced "pain"); placebo pellets or saline minipumps did not change thresholds. Opioid-induced pain was observed while morphine delivery continued and while the rats were not in withdrawal. RVM lidocaine, or bilateral lesions of the dorsolateral funiculus (DLF), did not change response thresholds in placebo-pelleted rats but blocked opioid-induced pain. The intrathecal morphine antinociceptive dose-response curve (DRC) in morphinepelleted rats was displaced to the right of that in placebopelleted rats, indicating antinociceptive "tolerance." RVM lido-
\end{abstract}

Opioid analgesics, especially morphine, continue as the primary treatment of moderate to severe pain. Numerous clinical case reports indicate that opioids can paradoxically produce abnormal pain, which includes allodynia (pain elicited by normally innocuous stimuli) and hyperalgesia (enhanced responses to noxious stimulation) (for review, see Arner et al., 1988). Typically, opioid-induced abnormal pain differs in location and quality from the original complaint (Ali, 1986; Stillman et al., 1987; De Conno et al., 1991; Devulder, 1997). Opioids can also elicit paradoxical, abnormal pain in experimental models after acute (Yaksh and Harty, 1988; Larcher et al., 1998; Celerier et al., 1999, 2000) or sustained (Mao et al., 1995) administration. The mechanisms of such opioid-induced paradoxical pain are unknown but have been linked in animals to the NMDA receptor (Mao et al., 1995; Larcher et al., 1998; Celerier et al., 1999, 2000).

In both clinical and experimental settings, sustained opioid analgesia is limited by tolerance. NMDA receptor blockade has been repeatedly demonstrated to block opioid tolerance because of either repeated or infused opioid administration (Trujillo and Akil, 1991; Tiseo and Inturrisi, 1993; Mao et al., 1994, 1995, 1996;

\footnotetext{
Received Sept. 1, 2000; revised Oct. 11, 2000; accepted Oct. 13, 2000.

Correspondence should be addressed to Dr. Frank Porreca, Department of Pharmacology, College of Medicine, University of Arizona Health Sciences Center, Tucson, AZ 85724. E-mail: frankp@u.arizona.edu.

Copyright (C) 2001 Society for Neuroscience 0270-6474/01/210279-08\$15.00/0
}

caine or bilateral DLF lesion did not alter the intrathecal morphine DRC in placebo-pelleted rats but blocked the rightward displacement seen in morphine-pelleted animals. The subcutaneous morphine antinociceptive DRC in morphinepelleted rats was displaced to the right of that in placebopelleted rats; this right shift was blocked by RVM lidocaine. The data show that (1) opioids elicit pain through tonic activation of bulbospinal facilitation from the RVM, (2) increased pain decreases spinal opioid antinociceptive potency, and (3) blockade of pain restores antinociceptive potency, revealing no change in antinociceptive signal transduction. These studies offer a mechanism for paradoxical opioid-induced pain and allow the development of approaches by which the loss of analgesic activity of opioids might be inhibited.

Key words: descending facilitation; opioid-induced pain; opioid tolerance; supraspinal/spinal synergy; lidocaine; tactile allodynia; thermal hyperalgesia

Tiseo et al., 1994; Manning et al., 1996). However, the mechanism by which opioid tolerance is altered by NMDA receptor blockade also remains unclear.

Fields et al. (1983), Fields and Heinricher (1985), Fields et al. (1991), and Morgan and Fields (1994) have characterized descending pain modulatory systems arising in the rostral ventromedial medulla (RVM). In these studies, bulbospinal projections mediating both descending pain inhibition and facilitation have been noted (for review, see Fields and Basbaum, 1999). Such descending pathways are believed to travel in the dorsolateral funiculus (DLF) to the spinal dorsal horn (Fields and Basbaum, 1999). Whereas the importance of descending pain facilitation under physiological conditions is unclear, the existence of these projections led us to hypothesize that possible tonic activation of such systems under pathological conditions may provide a mechanism for chronic abnormal pain such as that seen in neuropathic states (Kovelowski et al., 2000; Ossipov et al., 2000; Sun et al., 2000). The present studies have extended this hypothesis to examine whether tonic activation of descending pain facilitation may also provide a mechanism for the abnormal pain seen with sustained opioids. Our data suggest that the presence of opioidinduced pain resulting from sustained opioid delivery manifests behaviorally as antinociceptive tolerance. Blockade of such pain reveals no loss of opioid signal transduction. These findings open clinical possibilities by which opioid analgesia might be maintained in the treatment of chronic pain states. 


\section{MATERIALS AND METHODS}

Male Sprague Dawley rats (Harlan Sprague Dawley, Indianapolis, IN), 200-300 gm at time of testing, were maintained in a climate-controlled room on a $12 \mathrm{hr}$ light/dark cycle (lights on at 6:00 A.M.) with food and water available ad libitum. All testing was performed in accordance with the policies and recommendations of the International Association for the Study of Pain and the National Institutes of Health guidelines for the handling and use of laboratory animals and received approval from the Institutional Animal Care and Use Committee of the University of Arizona. It should be noted that term "pain" used in this report reflects measured changes in sensory thresholds (i.e., enhanced nociceptive responses) in animals. Any relationship to human states of pain are necessarily limited, and no claims are made regarding possible cortical or thalamic projections. Groups of 5-10 rats were used in all experiments.

Surgical procedures. While under halothane anesthesia, some groups of rats were implanted with intrathecal catheters $(\mathrm{PE}-10,7.5 \mathrm{~cm})$ as described previously (Yaksh and Rudy, 1976) for drug administration at the level of the lumbar spinal cord. Animals were allowed to recover for $4 \mathrm{~d}$.

Rats were prepared for bilateral RVM drug administration by placing anesthetized (ketamine-xylazine; $100 \mathrm{mg} / \mathrm{kg}$, i.p.) animals in a stereotaxic headholder. For intracranial bilateral drug administrations, the skull was exposed, and two 26 gauge guide cannula separated by $1.2 \mathrm{~mm}$ (Plastics One, Roanoke, VA) were directed toward the lateral portions of the RVM (anteroposterior $-11.0 \mathrm{~mm}$ from bregma, lateral $\pm 0.6 \mathrm{~mm}$ from midline, dorsoventral $-8.5 \mathrm{~mm}$ from the cranium); these coordinates were obtained from the atlas of Paxinos and Watson (1986). The guide cannulas were cemented in place and secured to the skull by small stainless steel machine screws. The animals were allowed to recover $5 \mathrm{~d}$ after surgery before any pharmacological manipulations were made. Drug administrations into the RVM were performed by slowly expelling $0.5 \mu \mathrm{l}$ of drug solution or saline through a 33 gauge injection cannula inserted through the guide cannula and protruding an additional $1 \mathrm{~mm}$ into fresh brain tissue to prevent backflow of drug into the guide cannula. At the termination of the experiments, Pontamine blue was injected into the site of the RVM injections, and catheter placement was verified histologically. Data from animals with incorrectly placed cannulas were discarded.

Spinal DLF lesions. Spinal lesions at the $\mathrm{T}_{8}$ level were performed in halothane-anesthetized rats. A laminectomy was made at the $\mathrm{T}_{8}$ level to expose the spinal cord. Lesions of the DLF were performed by crushing the area with fine forceps. Sham spinal surgery was performed by exposing the vertebrae and performing the laminectomy, but without cutting any neuronal tissue. Hemostasis was confirmed, and the wound over the exposed spinal cord was packed with Gelfoam and closed. All lesions were verified histologically at the termination of the experiment by fixing the spinal sections obtained from the lesion site in paraffin. Sections (40- $\mu \mathrm{m}$-thick) were mounted and stained with Luxor fast blue myelin stain to visualize intact and disrupted white matter. Behavioral results obtained only from animals that had appropriately placed DLF lesions were included in analysis.

Drug administration. For sustained administration, two $75 \mathrm{mg}$ morphine pellets or two placebo pellets were implanted subcutaneously. Continuous subcutaneous infusions were also performed with osmotic minipumps (Alza, Mountain View, CA). The osmotic pumps delivered saline at $1 \mu \mathrm{l} / \mathrm{hr}$ or morphine at $45 \mu \mathrm{g} \cdot \mu \mathrm{l}^{-1} \cdot \mathrm{hr}^{-1}$ for $7 \mathrm{~d}$ and were implanted in the subcutaneous space. Test compounds were injected either through the intrathecal catheter in a volume of $5 \mu \mathrm{l}$ followed by a $9 \mu \mathrm{l}$ saline flush or by a subcutaneous administration at a volume of 1 $\mathrm{ml} / \mathrm{kg}$. The intrathecal administration of morphine was performed 30 min before tail flick in all studies. Each animal was used only once to avoid possibility of acute tolerance. Lidocaine ( $4 \% \mathrm{w} / \mathrm{v})$ was administered bilaterally $(0.5 \mu \mathrm{l})$ into the RVM. In the tail flick, RVM lidocaine time course studies, lidocaine was either coadministered with morphine or given 10 or 20 min before or 10 or 20 min after morphine administration. Saline was injected into the RVM as a control for the lidocaine administration. Subcutaneous morphine or placebo pellets were implanted $2 \mathrm{~d}$ after animals underwent either sham or DLF lesion as described above.

Thermal hyperalgesia. The method of Hargreaves et al. (1988) was used to assess paw-withdrawal latency to a thermal nociceptive stimulus. Rats were allowed to acclimate within a Plexiglas enclosure on a clear glass plate maintained at $30^{\circ} \mathrm{C}$. A radiant heat source (i.e., high-intensity projector lamp) was activated with a timer and focused onto the plantar surface of the hindpaw. Paw-withdrawal latency was determined by a motion detector that halted both lamp and timer when the paw was withdrawn. The latency to withdrawal of the paw from the radiant heat source was determined both before and after drug or vehicle administration. Baseline latencies were established at $\sim 20 \mathrm{sec}$ to allow for detection of possible hyperalgesia. A maximal cutoff of $40 \mathrm{sec}$ was used to prevent tissue damage. Comparisons among group means were determined by Student's $t$ test at a significance level of 0.05 .

Tactile allodynia. The paw withdrawal thresholds of the hindpaws of the rats were determined in response to probing with eight calibrated von Frey filaments (Stoelting, Wood Dale, IL) in logarithmically spaced increments ranging from 0.41 to $15 \mathrm{gm}(4-150 \mathrm{mN})$. Each filament was applied perpendicularly to the plantar surface of the ligated paw of rats kept in suspended wire-mesh cages. Measurements were taken both before and after administration of drug or vehicle. Withdrawal threshold was determined by sequentially increasing and decreasing the stimulus strength ("up and down" method), analyzed using a Dixon nonparametric test (Chaplan et al., 1994) and expressed as the mean withdrawal threshold. Comparisons among group means were determined by Student's $t$ test at a significance level of 0.05 .

Tail-flick test. Acute nociception was determined by using the nociceptive hot water $\left(52^{\circ} \mathrm{C}\right)$ tail-flick reflex. The tail-flick test was performed by placing the distal third of the tail of rats in a heated water bath maintained at $52^{\circ} \mathrm{C}$. The latency until tail withdrawal (rapid flick) from the bath was determined and compared among the treatments. A $10 \mathrm{sec}$ cutoff was used to avoid tissue damage. Tolerance to the antinociceptive effect of opioids was indicated by a significant reduction in tail-flick latency after challenge with an $\mathrm{A}_{90}$ dose. Data were converted to percentage of antinociception by the following formula: (response latency baseline latency)/(cutoff - baseline latency) $\times 100$ to generate doseresponse curves (Tallarida and Murray, 1987). Regression analysis of the dose-response curves was used to detect significant shifts in drug potency (Tallarida and Murray, 1987).

Rotarod test. Rats were tested for their ability to balance on a slow rotating rod (diameter $3.5 \mathrm{~cm}$; rate of rotation $10 \mathrm{rpm}$ ). Rats were conditioned before the experiment, and rats (naive rats remaining on the rotarod for $180 \mathrm{sec}$ ) were then injected bilaterally (0.5:1) via the RVM route with either lidocaine $(4 \% \mathrm{w} / \mathrm{v})$ or saline. Rats were tested at $30 \mathrm{~min}$ after RVM administration, and the latency to fall off the rod was recorded. Animals falling of the rod before cutoff of $180 \mathrm{sec}$ were considered as having motor impairment.

Compounds. Morphine sulfate was purchased from Sigma (St. Louis, $\mathrm{MO})$. Morphine pellets and placebo pellets were a generous gift from the National Institute on Drug Abuse. Lidocaine (4\% w/v in sterile saline) was purchased from Roxane Laboratories (Columbus, OH). All compounds were dissolved in normal saline.

\section{RESULTS}

Sustained administration of morphine by subcutaneous implantation of two $75 \mathrm{mg}$ morphine pellets produced tactile allodynia and thermal hyperalgesia when measured on day 7 after pellet implantation. Paw withdrawal thresholds to probing with von Frey filaments were significantly reduced to $2.9 \pm 0.5 \mathrm{gm}$ from a baseline of $15 \pm 0 \mathrm{gm}$ (Fig. 1). Paw withdrawal latencies from radiant heat were significantly $(p \leq 0.05)$ reduced to $14.3 \pm 0.7$ $\mathrm{sec}$ from a baseline of $22.2 \pm 1.6 \mathrm{sec}$ (Fig. 1). Similar results were achieved with subcutaneous infusion of morphine through an osmotic pump (45 $\left.\mu \mathrm{g} \cdot \mu \mathrm{l}^{-1} \cdot \mathrm{hr}^{-1}\right)$ for $7 \mathrm{~d}$. Paw withdrawal thresholds were significantly $(p \leq 0.05)$ reduced from $15 \pm 0$ gm to $7.4 \pm 1.6 \mathrm{gm} 7 \mathrm{~d}$ after the start of morphine infusion. Similarly, paw withdrawal latencies were significantly $(p \leq 0.05)$ reduced from a preinfusion baseline of $22.0 \pm 0.6 \mathrm{sec}$ to $16.6 \pm 0.3 \mathrm{sec}$. The onset of tactile allodynia and thermal hyperalgesia elicited by subcutaneous morphine pellets were time-related and could be detected as early as day 2 (Fig. 2). As expected, antinociception was evident during the first few hours after subcutaneous morphine pellet implantation and could readily be detected in the foot-flick assay (Fig. 2). Placebo pellets did not elicit antinociceptive effects, and no changes in response thresholds to a nonnoxious stimulus were observed in placebo-pelleted animals (Fig. 2). Similarly, subcutaneous minipumps delivering saline did not 
A

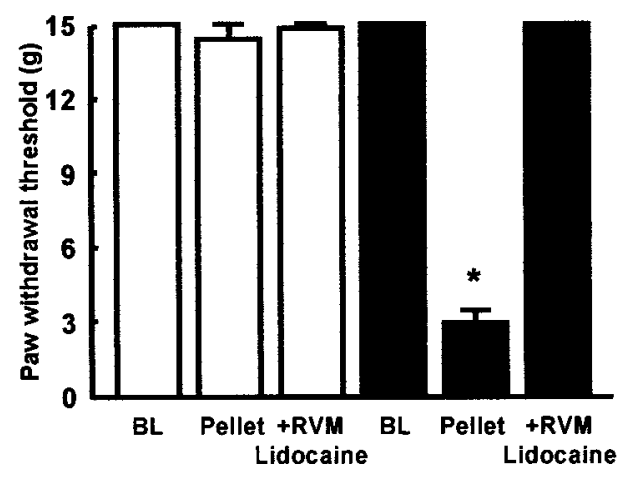

B

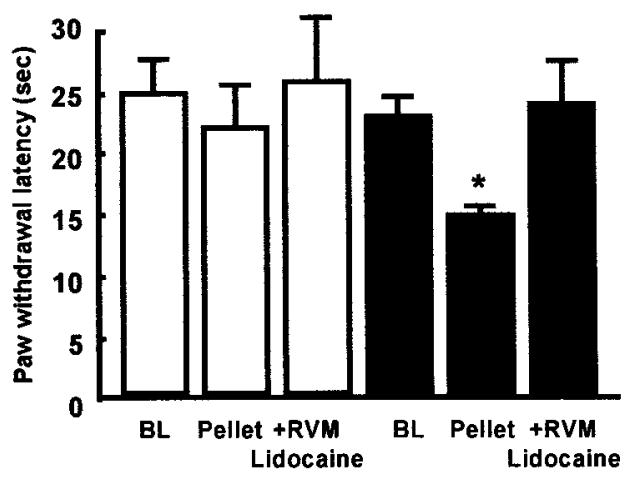

Figure 1. Male Sprague Dawley rats were implanted subcutaneously with either two placebo or two morphine (75 mg each) pellets. The $7 \mathrm{~d}$ of constant exposure to morphine pellets resulted in tactile allodynia indicated by a significant ( ${ }^{*} p \leq 0.05$; Student's $t$ test; $\left.n=10\right)$ decrease in paw withdrawal thresholds to probing with von Frey filaments $(A)$. The bilateral microinjection of lidocaine $(0.5 \mu \mathrm{l} ; 4 \% \mathrm{w} / \mathrm{v})$ into the RVM on day 7 blocked tactile allodynia when given 30 min before testing in morphine-pelleted rats $(n=10)(A)$. Rats with placebo pellets demonstrated no significant $(p>0.05)$ difference in paw withdrawal thresholds to probing with von Frey filaments on day 7 . The bilateral microinjection of lidocaine $(0.5 \mu \mathrm{l} ; 4 \%$ w/v) into the RVM on day 7 produced no changes in paw withdrawal thresholds in placebo-pelleted rats $(A)$. The exposure to subcutaneous morphine pellets also resulted in thermal hyperalgesia indicated by a significant $\left({ }^{*} p \leq 0.05\right.$; Student's $t$ test; $\left.n=10\right)$ decrease in paw withdrawal latencies to radiant heat applied to the plantar aspect of the hindpaw $(B)$. The bilateral microinjection of lidocaine $(0.5 \mu \mathrm{l} ; 4 \% \mathrm{w} / \mathrm{v})$ on day 7 blocked thermal hyperalgesia when given 30 min before testing in morphine-pelleted rats $(n=10)(B)$. Placebo-pelleted animals demonstrated no significant $(p>0.05)$ difference in paw withdrawal latencies to radiant heat on day 7 , nor were there any significant changes after the bilateral RVM administration of lidocaine ( $0.5 \mu \mathrm{l} ; 4 \% \mathrm{w} / \mathrm{v})$ $(B)$.
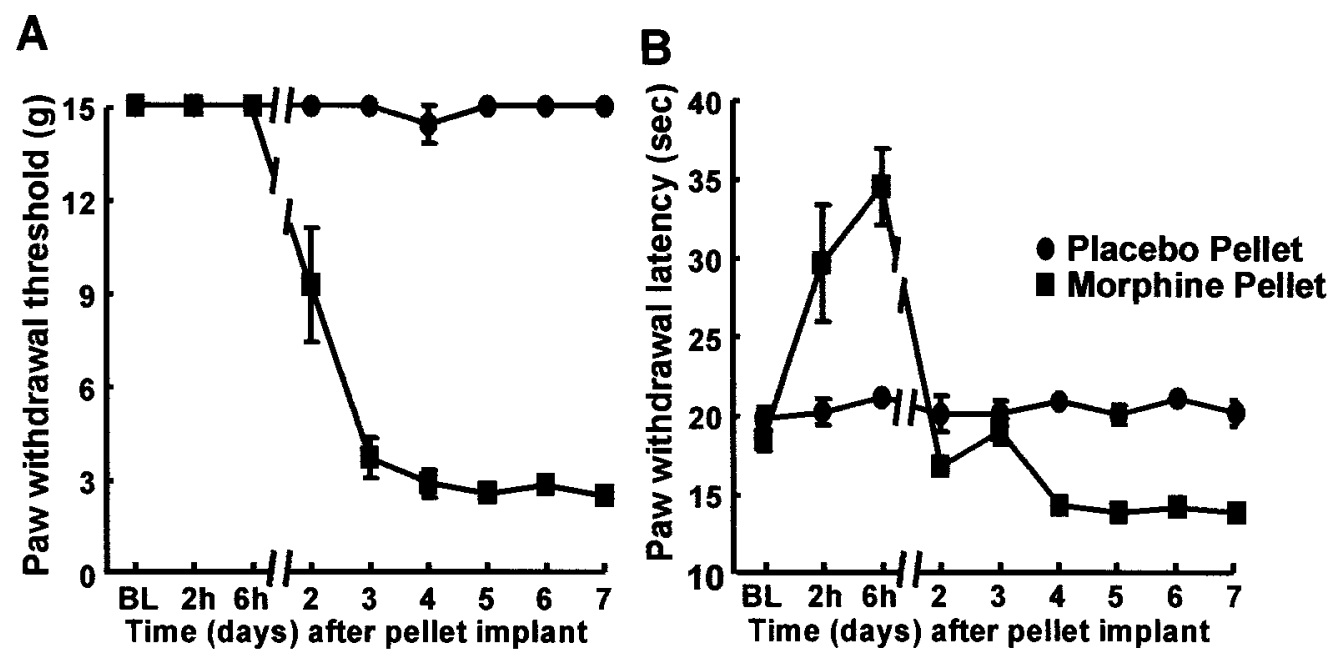

Figure 2. Male Sprague Dawley rats received subcutaneous implantation of placebo or morphine pellets (two $75 \mathrm{mg}$ pellets or $150 \mathrm{mg} / \mathrm{animal}$ ). Paw withdrawal thresholds to probing with von Frey filaments $(A)$ and paw withdrawal latencies to radiant heat $(B)$ applied to the plantar aspect of the hindpaw were determined 2 and $6 \mathrm{hr}$ after implantation and once daily afterward for $7 \mathrm{~d}$. Morphine pellets initially produced antinociception (at 2 and $6 \mathrm{hr}$ ) in the radiant heat paw-flick test $(B)$. Subsequently, tactile allodynia and thermal hyperalgesia, as indicated by a decrease in paw withdrawal thresholds and latencies were observed. Allodynia and hyperalgesia were significant $\left({ }^{*} p \leq 0.05\right.$; Student's $t$ test; $\left.n=6\right)$ by the second day after morphine pellet implantation.

elicit changes in sensory threshold to either non-noxious or noxious stimulation.

During measurement periods, both placebo- and morphinepelleted animals, and animals with osmotic minipumps delivering morphine or saline were carefully monitored for behavioral signs of opioid abstinence using a previously established scoring system. These included the presence of "wet-dog" shakes, excessive grooming, diarrhea, chromodacryorrhea, and jumping (Wei, 1973). In no cases were signs of opioid withdrawal observed over the $7 \mathrm{~d}$ period while morphine was being delivered; the behavioral scores for these withdrawal signs were essentially zero and showed no differences between placebo versus morphine-pelleted groups or saline- versus morphine-infusion groups (data not shown).

The administration of lidocaine $(0.5 \mu \mathrm{l}, 4 \% \mathrm{w} / \mathrm{v})$ into the RVM did not alter response thresholds to von Frey filaments or noxious thermal stimulation in placebo-pelleted or saline-infused rats. However, RVM lidocaine blocked both tactile allodynia and thermal hyperalgesia seen in morphine-pelleted or morphineinfused rats. Paw withdrawal thresholds to probing with von Frey filaments were increased to $15 \pm 0 \mathrm{gm}$ in both the morphine pellet-implanted (Fig. 1) and morphine-infused groups of rats. 

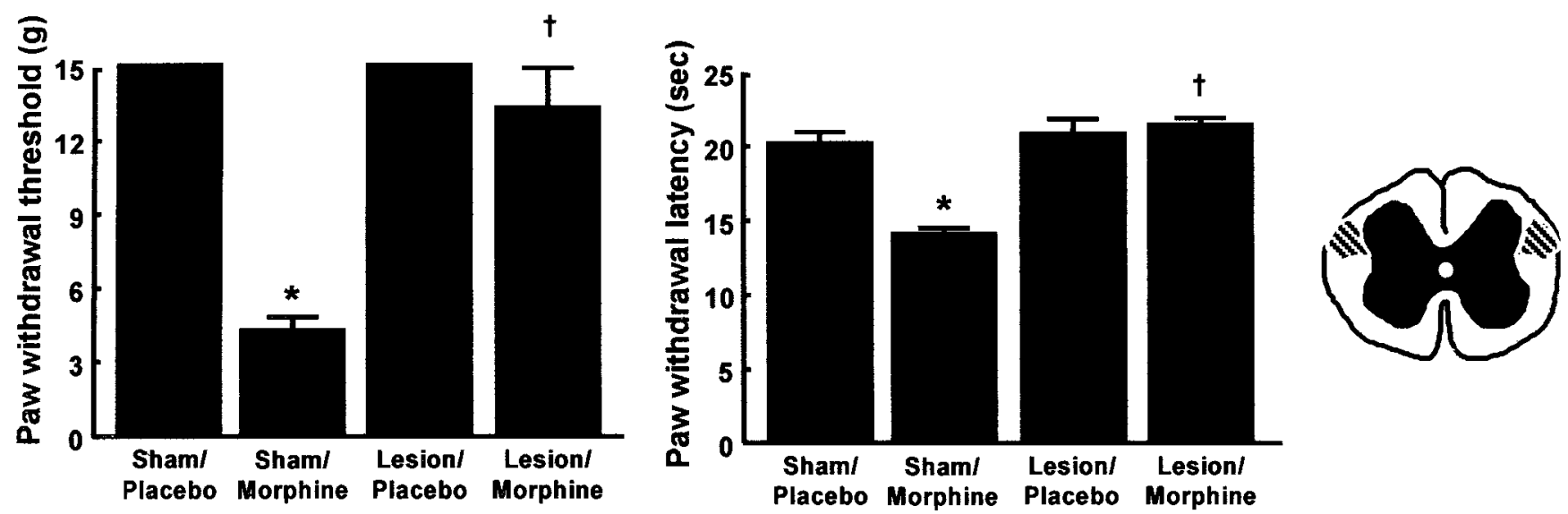

Figure 3. Male Sprague Dawley rats received either bilateral lesions of the DLF or sham lesions. In addition, $2 \mathrm{~d}$ after surgery, animals received either subcutaneous implantation of placebo pellets or morphine $(75 \mathrm{mg})$ pellets. No significant differences were observed in paw withdrawal thresholds to probing with von Frey filaments or to paw withdrawal latencies to radiant heat between animals receiving sham surgery and placebo pellets or DLF ablation and placebo pellets $(A)$. Morphine pellet implantation resulted in tactile allodynia, as indicated by a significant $\left({ }^{*} p \leq 0.05\right.$; Student's $t$ test; $n=$ 10) decrease in paw withdrawal thresholds to probing with von Frey filaments $(A)$, and thermal hyperalgesia, indicated by decreased paw withdrawal latencies to radiant heat $(B)$, in the rats with sham DLF lesions. However, morphine-induced tactile allodynia $(A)$ and thermal hyperalgesia $(B)$ both were completely blocked in animals with bilateral DLF lesions. The paw withdrawal thresholds to probing with von Frey filaments and paw and the withdrawal latencies to radiant heat were significantly ( $\dagger p \leq 0.05$; Student's $t$ test; $n=10$ ) greater than those of morphine-pelleted, sham-lesioned rats. $C$, A schematic of the cross-sectional view of the thoracic spinal cord. The shaded area depicts the area of bilateral DLF lesions.

Similarly, paw withdrawal latencies from a radiant heat source were increased by the microinjection of lidocaine into the RVM. The postlidocaine withdrawal latencies were $23.2 \pm 3.6$ and $21.1 \pm 1.3 \mathrm{sec}$ for the morphine pellet-implanted (Fig. 1) and inf used groups, respectively. These data indicate that RVM lidocaine returned sensory thresholds to, but not above, baseline levels. No behavioral abnormalities (ataxia, catatonia, loss of righting, or placement response) were noted in animals receiving RVM lidocaine. The microinjection of lidocaine into sites dorsal to the RVM, performed as a site control experiment $(n=4)$, produced motor hyperactivity including circling and barrelrolling behavior. No such signs were observed in rats with microinjections made into the RVM. The administration of saline in the RVM produced no changes in sensory thresholds or motor function. Neither lidocaine (4\% w/v) nor saline administered bilaterally (0.5:1) into the RVM impaired motor function. All animals remained on the rotarod until cutoff (data not shown).

In sham DLF-lesioned rats, subcutaneous morphine pellets elicited tactile allodynia and thermal hyperalgesia (measured on day 7 after morphine), as indicated by paw withdrawal thresholds to von Frey filaments of $4.2 \pm 0.7 \mathrm{gm}$ and paw withdrawal latencies to radiant heat of $13.9 \pm 0.7 \mathrm{sec}$ (Fig. 3). These values were significantly $(p \leq 0.05)$ less than those of sham-DLF rats implanted with placebo pellets. The corresponding values were $15 \pm 0$ gm and $20.6 \pm 1.2 \mathrm{sec}$, respectively (Fig. 3 ). In rats with DLF lesions, subcutaneous morphine pellet-induced allodynia and hyperalgesia were not observed. In these DLF-lesioned rats receiving subcutaneous morphine pellets, the paw withdrawal thresholds (measured on day 7 after morphine pellet) to von Frey filaments were $13.2 \pm 1.8 \mathrm{gm}$, and the paw withdrawal latencies to radiant heat were $21.2 \pm 0.7 \mathrm{sec}$ (Fig. 3). Lesions of the DLF did not change the behavioral responses of the placebo-implanted rats (Fig. 3).

Baseline tail-flick latencies were $3.9 \pm 0.14 \mathrm{sec}$ and $4.02 \pm 0.23$ sec in placebo and morphine-pelleted groups (measured on day 7 after pellet implantation), respectively, values that were not significantly different. The failure to detect hyperalgesia in the tail-flick response may reflect the rapid response latency in this endpoint. RVM lidocaine did not alter baseline tail-flick latencies in either placebo-pelleted or morphine-pelleted rats (data not shown). The subcutaneous implantation of morphine pellets for $7 \mathrm{~d}$ produced antinociceptive tolerance to intrathecal morphine. The antinociceptive dose-response for intrathecal morphine in rats with placebo pellets and bilateral administration of saline into the RVM in the $52^{\circ} \mathrm{C}$ tail flick test had an $A_{50}$ value of $1.4 \mu \mathrm{g}$ $[0.8-2.3$; 95\% confidence level (CL)]. The antinociceptive doseresponse for intrathecal morphine in morphine pellet-implanted rats and bilateral administration of saline into the RVM was significantly ( $p \leq 0.05$ ) shifted to the right, as indicated by an $A_{50}$ value of $35.0 \mu \mathrm{g}$ (26.3-46.8; 95\% CL) (Fig. 4). The concurrent microinjection of lidocaine $(0.5 \mu \mathrm{l} ; 4 \% \mathrm{w} / \mathrm{v})$ bilaterally into the RVM with intrathecal morphine restored the antinociceptive potency of morphine to that of the placebo-implanted rats (Fig. $4)$, as demonstrated by the $A_{50}$ value of $1.6 \mu \mathrm{g}(0.9-2.7 ; 95 \% \mathrm{CL})$. The microinjection of lidocaine into the RVM of the placebo pellet-implanted rats did not alter the antinociceptive potency of intrathecal morphine. The intrathecal morphine $A_{50}$ value was 1.7 $\mu \mathrm{g}(0.8-2.1 ; 95 \% \mathrm{CL})$ in the placebo pellet-implanted group in the presence of lidocaine; this value was not significantly different $(p>0.05)$ from that of placebo pellet alone (Fig. 4). The microinjection of saline into the RVM did not alter the antinociceptive effects of spinal morphine.

The effect of lidocaine in the RVM was short-acting and reversible. The greatest reduction in $A_{50}$ value in morphinetolerant rats occurred when lidocaine was microinjected concurrently with intrathecal morphine (Fig. 5). The microinjection of lidocaine either $20 \mathrm{~min}$ before or after intrathecal morphine yielded $A_{50}$ values of $29.0 \mu \mathrm{g}(20.6-40.9 ; 95 \% \mathrm{CL})$ and $31.2 \mu \mathrm{g}$ (17.5-55.8; 95\% CL), respectively. Likewise, the microinjection of lidocaine either $10 \mathrm{~min}$ before or after intrathecal morphine yielded $A_{50}$ values of $2.8 \mu \mathrm{g}(1.5-5.0 ; 95 \% \mathrm{CL})$ and $8.9 \mu \mathrm{g}$ (5.6-14.3; 95\% CL), respectively.

To further explore the possible bulbospinal contribution to behavioral manifestation of opioid antinociception, intrathecal 


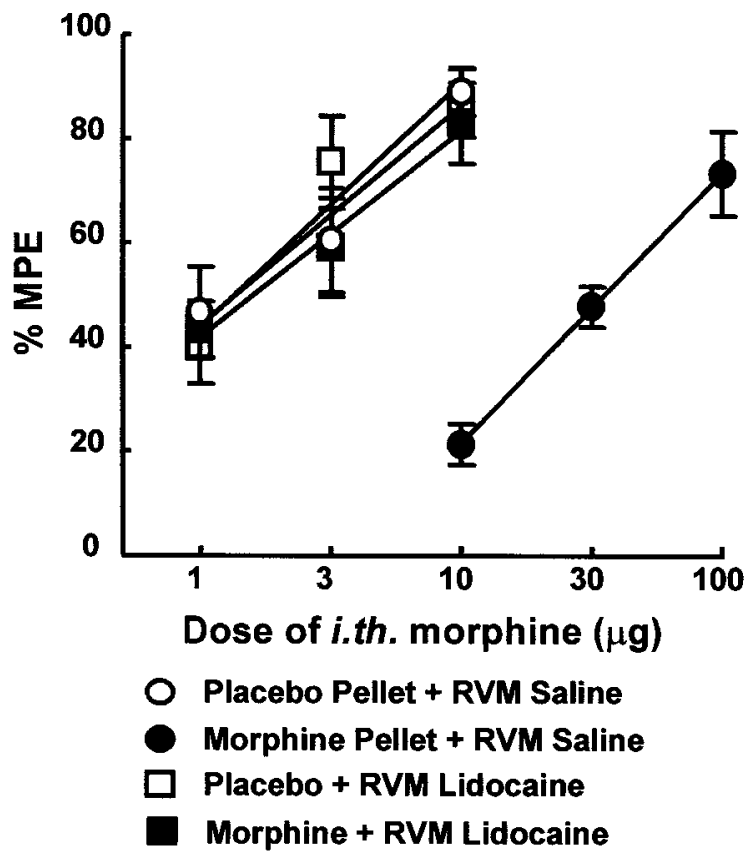

Figure 4. Male Sprague Dawley rats were implanted subcutaneous with either two placebo or two morphine ( $75 \mathrm{mg}$ each) pellets. Antinociceptive dose-response curves for intrathecal morphine were generated in the $52^{\circ} \mathrm{C}$ water tail flick test at the time of peak effect of morphine $(30 \mathrm{~min}$ as determined by pilot experiments). One group each of rats with placebo pellets and with morphine pellets received morphine intrathecally concurrently with bilateral RVM lidocaine $(0.5 \mu \mathrm{l} ; 4 \% \mathrm{w} / \mathrm{v})$. The following groups were used: placebo-pelleted rats with bilateral RVM saline and challenged with intrathecal morphine $(\bigcirc)$, placebo-pelleted rats with bilateral RVM lidocaine and challenged with intrathecal morphine ( $\square$ ), morphine-pelleted rats with bilateral RVM saline and challenged with intrathecal morphine ( $)$, and morphine-pelleted rats with bilateral RVM lidocaine and challenged with intrathecal morphine ( $\square$ ). The dose-effect curve for intrathecal morphine in the morphine-pelleted group was shifted significantly to the right of that for the placebo-pelleted group. This dose-effect curve was restored that of the placebo-pelleted groups by RVM lidocaine.

morphine dose-response curves were constructed using the $52^{\circ} \mathrm{C}$ tail-flick test in animals with either bilateral DLF or sham lesions in the presence of either morphine or placebo pellets. The antinociceptive $A_{50}$ values for intrathecal morphine in animals with placebo pellets and either DLF or sham lesions did not differ significantly ( $p \leq 0.05$ ); the $A_{50}$ values were $3.0 \mu \mathrm{g}(2.1-4.4 ; 95 \%$ CL) and $2.9 \mu \mathrm{g}(2.1-3.9 ; 95 \% \mathrm{CL})$, respectively. These values were also not significantly different from the $A_{50}$ value seen in unoperated group which was $3.38 \mu \mathrm{g}(2.0-3.7 \mu \mathrm{g}$; 95\% CL). The antinociceptive dose-response for intrathecal morphine in animals with sham-DLF lesion on day 7 of morphine pellet implantation was significantly ( $p \leq 0.05)$ shifted to the right, as indicated by an $A_{50}$ value of $19.8 \mu \mathrm{g}(16.0-24.4 ; 95 \%$ CL) (Fig. 6). This value did not differ significantly from that seen in unoperated rats implanted with morphine pellets in which the $A_{50}$ value was 21.6 $\mu \mathrm{g}$ (17.7-26.4 $\mu \mathrm{g} ; 95 \% \mathrm{CL})$. However, the intrathecal morphine dose-response in animals with DLF lesion and morphine pellets resulted in an $A_{50}$ value of $2.8 \mu \mathrm{g}(2.0-4.0 ; 95 \% \mathrm{CL}) 7 \mathrm{~d}$ after pellet implantation (Fig. 6). This dose-response curve was similar to those of the unoperated or the placebo-pelleted control groups.

Lidocaine in the RVM in the absence of subcutaneous morphine did not significantly change tail-flick baseline latencies in either placebo or morphine-pelleted rats (data not shown). The

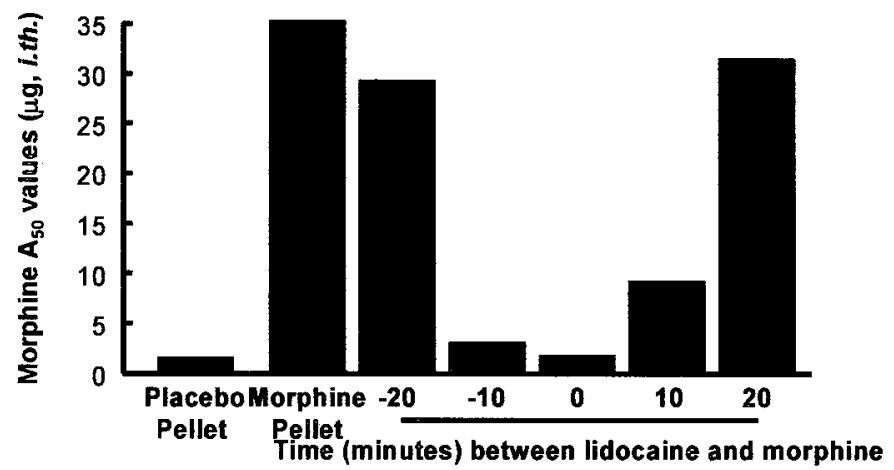

Figure 5. Male Sprague Dawley rats were implanted subcutaneously with either two placebo or two morphine $(75 \mathrm{mg}$ each) pellets. Antinociceptive dose-response functions for intrathecal morphine were generated in the $52^{\circ} \mathrm{C}$ water tail flick test at the time of peak effect of morphine $(30 \mathrm{~min})$. Lidocaine $(0.5 \mu \mathrm{l} ; 4 \% \mathrm{w} / \mathrm{v})$ was microinjected into the RVM at 0,10 , and $20 \mathrm{~min}$ before and after intrathecal morphine. The $A_{50}$ values were lowest, and similar to placebo-implanted values, when lidocaine was given concurrently with morphine. The largest $A_{50}$ values occurred when lidocaine was given $20 \mathrm{~min}$ before or after intrathecal morphine. These results demonstrate the reversibility of the effect of RVM lidocaine and suggest a direct causal relationship between inhibition of RVM activity and loss of morphine antinociceptive tolerance.
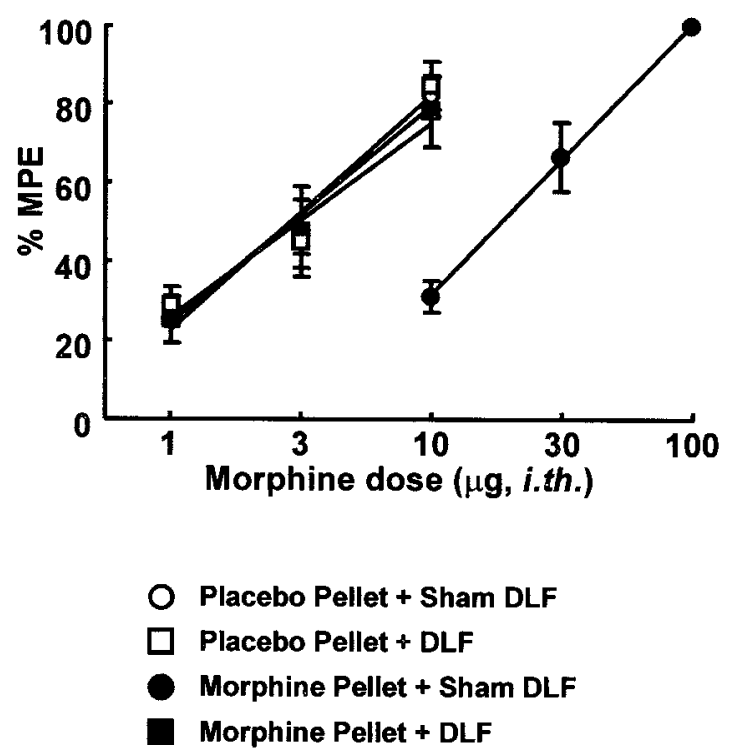

Figure 6. Male Sprague Dawley rats received either bilateral lesions of the DLF or sham lesions. In addition, $2 \mathrm{~d}$ after surgery, animals received either subcutaneous implantation of placebo pellets or morphine $(75 \mathrm{mg})$ pellets. After $7 \mathrm{~d}$ of pellet exposure, antinociceptive dose-response functions for intrathecal morphine were generated in the $52^{\circ} \mathrm{C}$ water tail flick test at the time of peak effect of morphine $(30 \mathrm{~min})$. The following groups were used: placebo-pelleted rats with sham DLF lesions $(\bigcirc)$, placebopelleted rats with DLF lesions $(\square)$, morphine-pelleted rats with sham DLF lesions (๑), and morphine-pelleted rats with DLF lesions (ם). The dose-effect curve for intrathecal morphine in the morphine-pelleted group was shifted significantly to the right of that for the placebo-pelleted group. This dose-effect curve of the morphine-pelleted group with DLF lesions was not different from that of the placebo-pelleted groups.

subcutaneous implantation of morphine pellets for $7 \mathrm{~d}$ also produced antinociceptive tolerance to subcutaneous morphine. The antinociceptive $A_{50}$ value for subcutaneous morphine in rats with placebo pellets in the $52^{\circ} \mathrm{C}$ tail flick test was $3.1 \mathrm{mg} / \mathrm{kg}(2.5-4.8$; $95 \%$ CL). The antinociceptive dose-response for subcutaneous 


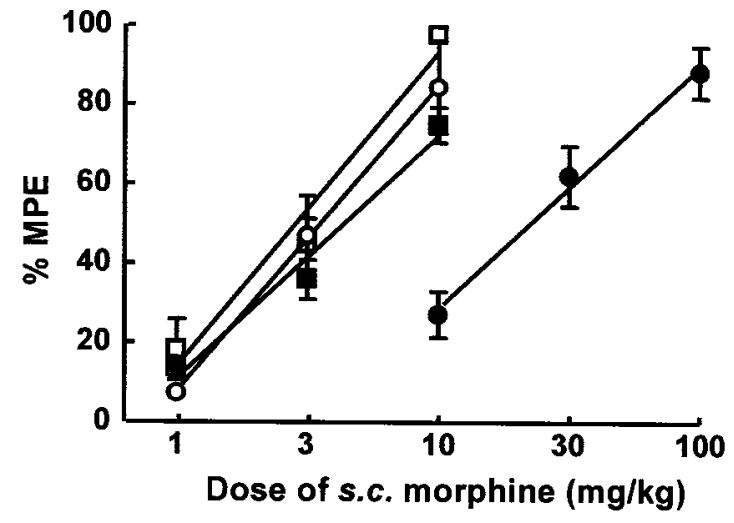

- Placebo Pellet + RVM Saline

$\square \quad$ Placebo Pellet + RVM Lidocaine

- MS Pellet + RVM Saline

- MS Pellet + RVM Lidocaine

Figure 7. Male Sprague Dawley rats were implanted subcutaneously with either two placebo or two morphine (75 mg each) pellets. Antinociceptive dose-response functions for subcutaneous morphine were generated in the $52^{\circ} \mathrm{C}$ water tail flick test at the time of peak effect of morphine $(30$ $\mathrm{min}$ ). One group each of rats with placebo pellets and with morphine pellets received morphine subcutaneously concurrently with bilateral RVM lidocaine $(0.5 \mu \mathrm{l} ; 4 \% \mathrm{w} / \mathrm{v})$. The following groups were used: placebo-pelleted rats with bilateral RVM saline and challenged with subcutaneous morphine $(\bigcirc)$, placebo-pelleted rats with bilateral RVM lidocaine and challenged with subcutaneous morphine ( $\square$ ), morphinepelleted rats with bilateral RVM saline and challenged with subcutaneous morphine (-), and morphine-pelleted rats with bilateral RVM lidocaine and challenged with subcutaneous morphine (ם). The dose-effect curve for subcutaneous morphine in the morphine-pelleted group was shifted significantly to the right of that for the placebo-pelleted group. This dose-effect curve was restored to that of the placebo-pelleted groups by RVM lidocaine.

morphine in the morphine pellet-implanted rats was significantly ( $p \leq 0.05$ ) displaced to the right, as indicated by an $A_{50}$ value of $21.6 \mathrm{mg} / \mathrm{kg}(15.7-29.6$; 95\% CL) (Fig. 7). The microinjection of lidocaine $(0.5 \mu \mathrm{l} ; 4 \% \mathrm{w} / \mathrm{v})$ bilaterally into the RVM concurrently with subcutaneous morphine restored the antinociceptive potency of morphine to that of the placebo-implanted rats (Fig. 7), as demonstrated by the $A_{50}$ value of $4.2 \mathrm{mg} / \mathrm{kg}(3.6-5.1 ; 95 \% \mathrm{CL})$. The microinjection of lidocaine into the RVM of the placebo pellet-implanted rats did not alter the antinociceptive potency of subcutaneous morphine. The subcutaneous morphine $A_{50}$ value of the placebo pellet-implanted group in the presence of lidocaine was $2.8 \mathrm{mg} / \mathrm{kg}(2.2-3.5 ; 95 \% \mathrm{CL})$, and it was not significantly ( $p>$ 0.05 ) different from that of placebo pellet alone (Fig. 7). Saline injected in the RVM did not alter the potency of subcutaneous morphine.

\section{DISCUSSION}

The present results suggest that sustained morphine exposure elicits neuroplasticity resulting in tonic activation of descending facilitation. The importance of the RVM to the neuroplastic changes resulting in pain caused by tonic facilitation is demonstrated by (1) the time-related onset of opioid-induced pain and (2) the reversible blockade of opioid-induced pain by RVM lidocaine. Disruption of the DLF, a principal neural conduit of spinopetal tracts from the RVM, including those mediating facilitation of pain, blocked opioid-induced pain without altering normal sensory thresholds or motor activity. Importantly, opioidinduced pain was evident during the course of morphine delivery by continuous infusion with osmotic minipumps, as well as by morphine pellets, minimizing possible pharmacokinetic concerns about sustained opioid delivery. No signs of the opioid withdrawal syndrome were detected in spite of careful monitoring, suggesting that opioid-induced pain observed was not the result of abstinence or states of "mini-withdrawal"(Gutstein, 1996). This conclusion is reinforced by the time-related onset of opioid-induced pain, which was clearly detectable by day 3 of morphine administration. The consequence of opioid-induced pain was significant in determining subsequent opioid antinociceptive potency. Our studies show that manipulations that block opioid-induced pain, such as RVM lidocaine or DLF lesions, also restore morphine antinociceptive potency. These manipulations revealed no apparent alteration of antinociceptive signal transduction in morphinepelleted rats. Based on such observations, the central hypothesis of this investigation is that tonic activation of descending facilitation arising in the RVM represents a mechanism of chronic pain, including that induced by opioids, and that such opioidinduced pain manifests behaviorally as antinociceptive tolerance.

Some studies have reported opioid-induced hyperalgesia (Colpaert, 1996; Laulin et al., 1999) although others did not find this effect (Kayser and Guilbaud, 1985; Gutstein et al., 1995). Reported "opioid-induced" hyperalgesia has been suggested to result from the unmasking of compensatory neuronal hyperactivity occurring after the opioid is removed or intermittently between injections (Gutstein, 1996). Whereas the nature of such neuronal hyperactivity remains to be elucidated, the resulting hyperalgesia could be interpreted as the result of episodes of opioid "miniwithdrawals" (Gutstein, 1996). This possibility may apply to studies of opioid hyperalgesia conducted either after termination of opioid administration or with repeated injections (Trujillo and Akil, 1991; Mao et al., 1994, 1995; Larcher et al., 1998; Laulin et al., 1998; Celerier et al., 2000).

The present studies with sustained morphine differ from these previous investigations in several important ways. First, our data show that sustained opioid administration elicits not only thermal hyperalgesia but tactile allodynia as well. The latter is a manifestation of an abnormal pain state, in which sustained opioid exposure resulted in normally non-noxious light touch producing apparent pain. Clinical reports exist of patients developing abnormal pain including both allodynia and hyperalgesia developed while patients were receiving opioids (Arner et al., 1988) and may be different in location and quality than the original complaint (Ali, 1986; Stillman et al., 1987; Devulder, 1997). Second, these behavioral signs of abnormal pain were not immediate, but developed over a period of days, suggesting the need for neuronal plasticity. Third, allodynia and hyperalgesia developed and were maintained during continuous morphine delivery caused by either pellets or osmotic minipump. These similar results in spite of differences in delivery systems minimize pharmacokinetic concerns and suggest that the possibility that observed hyperesthesias might be attributable to opioid withdrawal is unlikely. Indeed, no signs of withdrawal were observed despite careful monitoring.

Descending pain modulatory influences, including facilitation, have been identified in the RVM (Zhuo and Gebhart, 1992; Urban et al., 1996; Urban and Gebhart, 1997; Fields and Basbaum, 1999). RVM neuronal populations include "OFF" cells that comprise a descending inhibitory system and "ON" cells that facilitate nociceptive processing through spinopetal fibers (Fields 
et al., 1983, 1991; Fields and Heinricher, 1985). The existence of descending facilitation is supported by observations that prolonged stimulation with noxious heat increased ON-cell and decreased OFF-cell activity and enhanced nociceptive responses (Morgan and Fields, 1994). ON-cell activity has been found to increase along with heightened nociception during naloxoneprecipitated withdrawal (Bederson et al., 1990; Kim et al., 1990), which were blocked by lidocaine in the RVM (Kaplan and Fields, 1991). However, the state of ON-cell or OFF-cell firing during sustained morphine administration, in the absence of withdrawal, has not been studied. The presence of such descending pain facilitation systems in the RVM led us to hypothesize that opioidinduced pain might be the consequence of inappropriate tonic descending facilitation, possibly because of increased ON-cell discharge. Our data support this possibility. Tonic neuronal discharge in the RVM is supported by our findings of blockade of opioid-induced pain by microinjection of lidocaine into this site. This hypothesis is further strengthened by the fact that spinopetal projections are known to descend through the DLF (Fields and Basbaum, 1999) and our observations in the present studies that DLF lesions also blocked opioid-induced pain.

Our studies also show an intimate relationship between the presence of opioid-induced pain and the antinociceptive potency of morphine. Manipulations that blocked opioid-induced enhanced pain, such as RVM microinjection of lidocaine or DLF lesion, also restored the spinal morphine antinociceptive potency. The specificity of the observed effects is reinforced by (1) the time-related restoration of spinal morphine potency by RVM lidocaine and (2) the site-specificity of lidocaine administration. Additionally, neither RV M lidocaine or DLF lesion altered baseline sensory thresholds, motor activity, or the spinal morphine dose-effect curve in placebo-implanted rats, indicating no tonic activity of RVM systems under normal conditions and support the view of time-related RVM plasticity to initiate descending facilitation, opioid-induced abnormal pain, and subsequent decreased spinal antinociceptive potency of morphine. These data reasonably suggest that spinal morphine antinociceptive potency depends on the intrinsic baseline nociceptive state and that the observed spinal potency of morphine reflects this pain state in animals as it does clinically (Cherney and Portenoy, 1999).

Systemic morphine antinociceptive potency is known to depend on antinociceptive synergy between spinal and supraspinal actions (Yeung and Rudy, 1980; Roerig and Fujimoto, 1989). This site-site synergy may be an important factor in the clinical potency and ultimate utility of this molecule. An important feature of opioid tolerance is the loss of antinociceptive spinal/ supraspinal synergy (Roerig et al., 1984; Roerig and Fujimoto, 1988). It seems reasonable to suggest that the observed decreased antinociceptive potency of systemic morphine in morphine pelletimplanted rats results from a loss of supraspinal-spinal antinociceptive synergy and that an important contributor to such lost synergy is the decreased spinal morphine potency in these rats. Our finding that RVM lidocaine restored systemic morphine antinociceptive potency might be interpreted as resulting from the restoring supraspinal-spinal synergy subsequent to normalizing spinal morphine activity caused by blockade of descending facilitation. Isobolographic analysis will be required to confirm this idea.

It has been determined that disinhibition of OFF cells is the principal mechanism of opioid-mediated antinociception in the RVM (Heinricher et al., 1994). In the present study, systemic morphine was active in both the morphine- or placebo-pelleted groups after RVM lidocaine. Because lidocaine would be expected to inhibit essentially all RVM neuronal activity, it seems likely that the disinhibition of RVM OFF cells may not be solely responsible for supraspinal opioid-mediated antinociception. Many supraspinal sites that mediate antinociception bypass the RVM either partly or completely (for review, see Fields and Basbaum, 1999). For example, the periaqueductal gray (PAG), a major source of projections to the RVM and of opioid-mediate antinociception, also sends some projections directly to the spinal cord (Castiglioni et al., 1978; Willis and Westlund, 1997). Furthermore, the PAG has neuronal communications with noradrenergic nuclei, including the locus coeruleus, A5, and A7, and these regions also have direct spinopetal projections (for review, see Fields and Basbaum, 1999). Such projections may be sufficient to reinstate hypothesized supraspinal-spinal opioid synergy subsequent to restoration of spinal morphine antinociceptive potency in morphine-pelleted rats after RVM lidocaine. Further experimental data will be required to substantiate this concept.

The results of the present investigation support the hypothesis that tonic descending facilitation may be an important mechanism of chronic pain and may underlie not only pain from sustained opioids but other conditions as well. In this regard, the abnormal pain elicited by experimental spinal nerve injury is also blocked by RVM lidocaine (Kovelowski et al., 2000). Additionally, experimental neuropathic pain is associated with decreased spinal morphine antinociceptive potency (Bian et al., 1999), which is restored by RVM lidocaine (Kovelowski et al., 2000). Additionally, DLF lesions also blocked nerve injury-induced pain (Ossipov et al., 2000). Taken together, these data support the view that abnormal pain caused by tonic descending facilitation from the RVM decreases the antinociceptive potency of spinal (and systemic) morphine. Blockade of the pain restores spinal morphine potency and may reinstate supraspinal-spinal opioid antinociceptive synergy after sustained opioid exposure or resulting from nerve injury (Bian et al., 1999). The decrease in spinal opioid antinociceptive potency seen under conditions of sustained opioids or nerve injury may therefore underlie the apparent loss of opioid analgesia (i.e., tolerance) seen clinically with opioid use for chronic pain or the limited opioid activity seen in neuropathic pain patients. Our data show no apparent change in antinociceptive signal transduction after blockade of opioid-induced pain. It seems likely that approaches that may block the RVM plasticity associated with sustained opioid exposure (or nerve injury) may allow sustained analgesic efficacy of opioids and their use in treatment of chronic pain.

\section{REFERENCES}

Ali NM (1986) Hyperalgesic response in a patient receiving high concentrations of spinal morphine [letter]. Anesthesiology 65:449.

Arner S, Rawal N, Gustafsson LL (1988) Clinical experience of longterm treatment with epidural and intrathecal opioids-a nationwide survey. Acta Anaesthesiol Scand 32:253-259.

Bederson JB, Fields HL, Barbaro NM (1990) Hyperalgesia during naloxone-precipitated withdrawal from morphine is associated with increased on-cell activity in the rostral ventromedial medulla. Somatosens Mot Res 7:185-203.

Bian D, Ossipov MH, Ibrahim M, Raffa RB, Tallarida RJ, Malan Jr TP, Lai J, Porreca F (1999) Loss of antiallodynic and antinociceptive spinal/supraspinal morphine synergy in nerve-injured rats: restoration by MK-801 or dynorphin antiserum. Brain Res 831:55-63.

Castiglioni AJ, Gallaway MC, Coulter JD (1978) Spinal projections from the midbrain in monkey. J Comp Neurol 178:329-346.

Celerier E, Laulin J, Larcher A, Le Moal M, Simonnet G (1999) Evidence for opiate-activated NMDA processes masking opiate analgesia in rats. Brain Res 847:18-25.

Celerier E, Rivat C, Jun Y, Laulin JP, Larcher A, Reynier P, Simonnet 
G (2000) Long-lasting hyperalgesia induced by fentanyl in rats: preventive effect of ketamine [see comments]. Anesthesiology 92:465-472.

Chaplan SR, Bach FW, Pogrel JW, Chung JM, Yaksh TL (1994) Quantitative assessment of tactile allodynia in the rat paw. J Neurosci Methods 53:55-63.

Cherney NI, Portenoy RK (1999) Practical issues in the management of cancer pain. In: Textbook of pain, Ed 4 (Wall PD, Melzack R, eds), pp 1479-1522. Edinburgh: Churchill Livingstone.

Colpaert FC (1996) System theory of pain and of opiate analgesia: no tolerance to opiates. Pharmacol Rev 48:355-402.

De Conno F, Caraceni A, Martini C, Spoldi E, Salvetti M, Ventafridda V (1991) Hyperalgesia and myoclonus with intrathecal infusion of highdose morphine. Pain 47:337-339.

Devulder J (1997) Hyperalgesia induced by high-dose intrathecal sufentanil in neuropathic pain. J Neurosurg Anesthesiol 9:146-148.

Fields HL, Basbaum AI (1999) Central nervous system mechanisms of pain modulation. In: Textbook of pain, Ed 4 Edition (Wall PD, Melzack R, eds), pp 309-329. Edinburgh: Churchill Livingstone.

Fields HL, Bry J, Hentall I, Zorman G (1983) The activity of neurons in the rostral medulla of the rat during withdrawal from noxious heat. J Neurosci 3:2545-2552.

Fields HL, Heinricher MM (1985) Anatomy and physiology of a nociceptive modulatory system. Philos Trans R Soc Lond B Biol Sci 308:361-374.

Fields HL, Heinricher MM, Mason P (1991) Neurotransmitters in nociceptive modulatory circuits. Annu Rev Neurosci 14:219-245.

Gutstein HB (1996) The effects of pain on opioid tolerance: how do we resolve the controversy? Pharmacol Rev 48:403-407 [discussion 409-411].

Gutstein HB, Trujillo KA, Akil H (1995) Does chronic nociceptive stimulation alter the development of morphine tolerance? Brain Res 680:173-179.

Hargreaves K, Dubner R, Brown F, Flores C, Joris J (1988) A new and sensitive method for measuring thermal nociception in cutaneous hyperalgesia. Pain 32:77-88.

Heinricher MM, Morgan MM, Tortorici V, Fields HL (1994) Disinhibition of off-cells and antinociception produced by an opioid action within the rostral ventromedial medulla. Neuroscience 63:279-288.

Kaplan H, Fields HL (1991) Hyperalgesia during acute opioid abstinence: evidence for a nociceptive facilitating function of the rostral ventromedial medulla. J Neurosci 11:1433-1439.

Kayser V, Guilbaud G (1985) Can tolerance to morphine be induced in arthritic rats? Brain Res 334:335-338.

Kim DH, Fields HL, Barbaro NM (1990) Morphine analgesia and acute physical dependence: rapid onset of two opposing, dose-related processes. Brain Res 516:37-40.

Kovelowski CJ, Ossipov MH, Sun H, Lai J, Malan TP, Porreca F (2000) Supraspinal cholecystokinin may drive tonic descending facilitation mechanisms to maintain neuropathic pain in the rat. Pain 87:265-273.

Larcher A, Laulin JP, Celerier E, Le Moal M, Simonnet G (1998) Acute tolerance associated with a single opiate administration: involvement of $N$-methyl-D-aspartate-dependent pain facilitatory systems. Neuroscience 84:583-589.

Laulin JP, Larcher A, Celerier E, Le Moal M, Simonnet G (1998) Long-lasting increased pain sensitivity in rat following exposure to heroin for the first time. Eur J Neurosci 10:782-785.

Laulin JP, Celerier E, Larcher A, Le Moal M, Simonnet G (1999) Opiate tolerance to daily heroin administration: an apparent phenomenon associated with enhanced pain sensitivity. Neuroscience 89:631636.

Manning BH, Mao J, Frenk H, Price DD, Mayer DJ (1996) Continuous co-administration of dextromethorphan or MK-801 with morphine: attenuation of morphine dependence and naloxone-reversible attenuation of morphine tolerance. Pain 67:79-88.

Mao J, Price DD, Mayer DJ (1994) Thermal hyperalgesia in association with the development of morphine tolerance in rats: roles of excitatory amino acid receptors and protein kinase C. J Neurosci 14:2301-2312.

Mao J, Price DD, Mayer DJ (1995) Mechanisms of hyperalgesia and morphine tolerance: a current view of their possible interactions. Pain 62:259-274.

Mao J, Price DD, Caruso FS, Mayer DJ (1996) Oral administration of dextromethorphan prevents the development of morphine tolerance and dependence in rats. Pain 67:361-368.

Morgan MM, Fields HL (1994) Pronounced changes in the activity of nociceptive modulatory neurons in the rostral ventromedial medulla in response to prolonged thermal noxious stimuli. J Neurophysiol 72:1161-1170.

Ossipov MH, Sun H, Malan TP, Lai J, Porreca F (2000) Mediation of spinal nerve injury induced tactile allodynia by descending facilitatory pathways in the dorsolateral funiculus. Neurosci Lett 290:129-132.

Paxinos G, Watson C (1986) The rat brain in stereotaxic coordinates, Ed 2. San Diego: Academic.

Roerig SC, Fujimoto JM (1988) Morphine antinociception in different strains of mice: relationship of supraspinal-spinal multiplicative interaction to tolerance. J Pharmacol Exp Ther 247:603-608.

Roerig SC, Fujimoto JM (1989) Multiplicative interaction between intrathecally and intracerebroventricularly administered mu opioid agonists but limited interactions between delta and kappa agonists for antinociception in mice. J Pharmacol Exp Ther 249:762-768.

Roerig SC, O'Brien SM, Fujimoto JM, Wilcox GL (1984) Tolerance to morphine analgesia: decreased multiplicative interaction between spinal and supraspinal sites. Brain Res 308:360-363.

Stillman MJ, Moulin DE, Foley KM (1987) Paradoxical pain following high-dose spinal morphine. Pain 4.

Sun H, Ren K, Zhong CM, Ossipov MH, Malan TP, Lai J, Porreca F (2000) Nerve-injury induced tactile allodynia is mediated via ascending spinal dorsal column projections. Pain, in press.

Tallarida RJ, Murray RB (1987) Manual of pharmacologic calculations with computer programs, 2 Ed. New York: Springer.

Tiseo PJ, Inturrisi CE (1993) Attenuation and reversal of morphine tolerance by the competitive $N$-methyl-D-aspartate receptor antagonist, LY274614. J Pharmacol Exp Ther 264:1090-1096.

Tiseo PJ, Cheng J, Pasternak GW, Inturrisi CE (1994) Modulation of morphine tolerance by the competitive $N$-methyl-D-aspartate receptor antagonist LY274614: assessment of opioid receptor changes. J Pharmacol Exp Ther 268:195-201.

Trujillo KA, Akil H (1991) Inhibition of morphine tolerance and dependence by the NMDA receptor antagonist MK-801. Science 251:85-87.

Urban MO, Gebhart GF (1997) Characterization of biphasic modulation of spinal nociceptive transmission by neurotensin in the rat rostral ventromedial medulla. J Neurophysiol 78:1550-1562.

Urban MO, Jiang MC, Gebhart GF (1996) Participation of central descending nociceptive facilitatory systems in secondary hyperalgesia produced by mustard oil. Brain Res 737:83-91.

Wei E (1973) Assessment of precipitated abstinence in morphinedependent rats. Psychopharmacologia 28:35-44.

Willis WD, Westlund KN (1997) Neuroanatomy of the pain system and of the pathways that modulate pain. J Clin Neurophysiol 14:2-31.

Yaksh TL, Harty GJ (1988) Pharmacology of the allodynia in rats evoked by high dose intrathecal morphine. J Pharmacol Exp Ther 244:501-507.

Yaksh TL, Rudy TA (1976) Chronic catheterization of the spinal subarachnoid space. Physiol Behav 17:1031-1036.

Yeung JC, Rudy TA (1980) Multiplicative interaction between narcotic agonisms expressed at spinal and supraspinal sites of antinociceptive action as revealed by concurrent intrathecal and intracerebroventricular injections of morphine. J Pharmacol Exp Ther 215:633-642.

Zhuo M, Gebhart GF (1992) Characterization of descending facilitation and inhibition of spinal nociceptive transmission from the nuclei reticularis gigantocellularis and gigantocellularis pars alpha in the rat. J Neurophysiol 67:1599-1614. 